\title{
Phosphorescence based oxygen sensors - essential tools for cell biology and life science research
}

\author{
Dmitri B. Papkovsky ${ }^{1}$ \\ ${ }^{1}$ School of Biochemistry and Cell Biology, University College Cork, Cork, Ireland \\ d.papkovsky@ucc.ie
}

\begin{abstract}
:
Various in vitro, ex-vivo and in vivo cell and tissue models are currently used in life science research, however for many of them control of sample oxygenation and cellular $\mathrm{O}_{2}$ levels is inadequate. Since $\mathrm{O}_{2}$ is a key parameter and informative biomarker of cellular function, knowledge of actual $\mathrm{O}_{2}$ levels in different compartments of biological samples and implementation of reliable in situ control of cellular $\mathrm{O}_{2}$ are of paramount importance. Phosphorescence based $\mathrm{O}_{2}$ sensors provide such capabilities and enable many important bioanalytical tasks with complex biological samples. Here I describe the spectrum of $\mathrm{O}_{2}$ sensing platforms developed in recent years by us and other research groups. These systems operate with solid-state sensors, soluble probes and imaging nanosensors, in conjunction with portable handheld instruments, commercial plate readers and sophisticated live cell imaging detection platforms. Analytical performance and practical uses of these sensor systems will be demonstrated in examples of physiological studies performed with simple 2D cell models, more complex multicellular spheroids, hetero-cellular organoids and cultured tissue slices. Integration of these tools in the current paradigm of life science research will also be discussed.
\end{abstract}

Key words: Optical oxygen sensors, phosphorescence quenching, intracellular, extracellular and imaging probes, cell and tissue oxygenation, metabolism, 3D tissue models

\section{Introduction}

Control of oxygen concentration and oxygenation conditions in cell and tissue culture is necessary at all levels. Macroscopic control is required for gaseous $\mathrm{O}_{2}$ in culturing chamber, especially in hypoxia workstations, and for dissolved $\mathrm{O}_{2}$ in growth media used to periodically feed the cells (it is usually oversaturated with $\mathrm{O}_{2}$ when taken from a fridge or ambient environment). Microscopic control or measurement of dissolved $\mathrm{O}_{2}$ is required when analyzing respiration, metabolism of small biological samples and various bioassays with live cells. Finally, quantitative measurements and monitoring of in situ oxygenation of cultured cells (average $\mathrm{O}_{2}$ for the monolayer), intracellular oxygen levels in individual cells and in highly heterogeneous 3D models of mammalian tissue, sub-cellular and tissue oxygen gradients - is of high value for complex physiological studies [1].

Phosphorescence-based $\mathrm{O}_{2}$ sensors and analytical platforms on their basis provide such capabilities, along with high versatility and convenience. They enable facile, quantitative and accurate, non- or minimally invasive measurements and continuous monitoring of $\mathrm{O}_{2}$ concentration in various biological samples and biomedical applications [2]. These systems are gaining popularity and many cell biology labs start using them routinely in their studies with live cell and tissue models. A number of such systems are now produced commercially. They are often tailored to perform specific analytical tasks, but at the same time offer flexibility and suitability for many new applications.

\section{Main Types of $\mathrm{O}_{2}$ Sensor Materials and Detection Instrumentation}

To date, a number of different sensor formats and detection platforms have been developed particularly for contact-less sensing of $\mathrm{O}_{2}$ in biological samples and for the implementation of the above mentioned analytical and biological tasks [3]. Some of the common modifications are shown in Fig. 1. The main types of sensor materials include solid-state sensors in the form of adhesive stickers or standalone bead sensors (Fig. 1A,B) as well as thin film coatings on assay vessels, such as microtitter plates, and fibre-optic micro-sensors. These sensors usually produce high phosphorescent signals and allow facile point measurements $\mathrm{O}_{2}$ concentration in macroscopic samples (also in 
microscopic samples with fibre-optic micro-sensors), with a simple LED-photodiode based handheld reader (Fig. 1D).

A

D

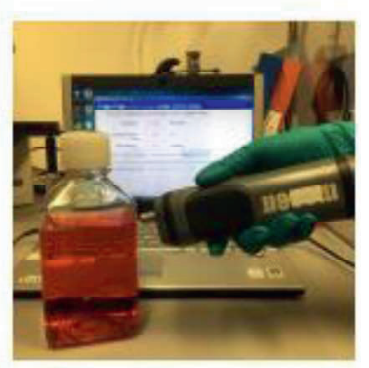

C

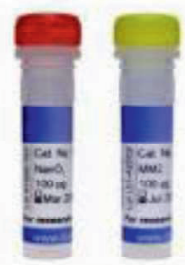

$\mathrm{E}$

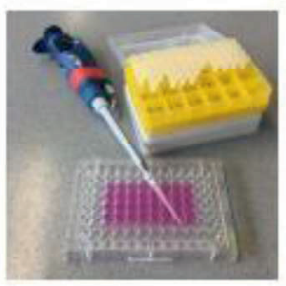

$\mathrm{F}$
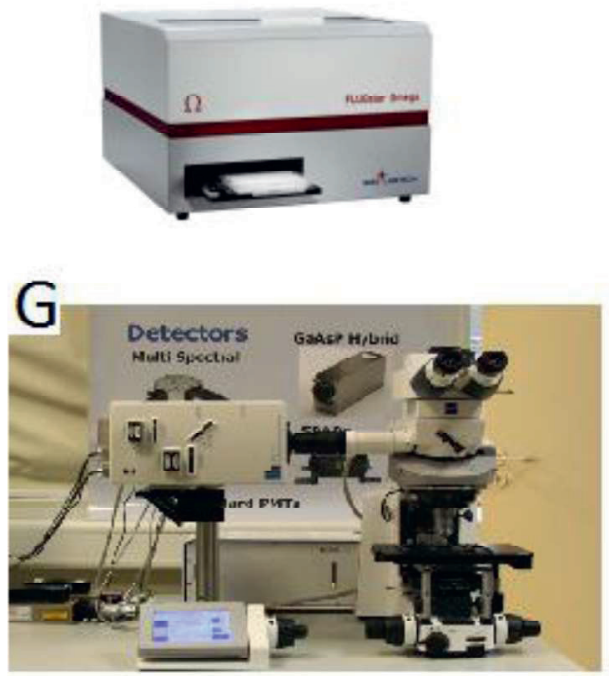

Fig.1. Examples of solid-state $\mathrm{O}_{2}$ sensors (sticker - $A$ and bead - $B$ ) and soluble probes $(C-$ extracellular or intracellular), their practical use/handling $(D, E)$ and detection instrumentation available (handheld reader $-D$, TRF plate reader - F, PLIM microscope - G).

Another type is soluble $\mathrm{O}_{2}$-sensing probes - liquid reagents that can be dispensed with a micropipette to various samples or assay substrates (Fig. 1E). One sub-group here is cell-impermeable (or extracellular) probes which stay in the medium and inform on $\mathrm{O}_{2}$ concentration and its changes in the bulk of the sample. Another sub-group is cell-permeable probes which have the ability to penetrate cells, accumulate and stay there and thus report on in situ or intracellular $\mathrm{O}_{2}$. Such $\mathrm{O}_{2}$ probes can comprise small molecule derivatives of phosphorescent $\mathrm{O}_{2}$-sensitive dyes, dyes conjugated to a suitable carrier such as a macromolecule, dendrimer or nanoparticle structure. The most common phosphorescent reporter dyes in $\mathrm{O}_{2}$ sensors and probes are $\mathrm{Pt}(\mathrm{II})$-complexes of porphyrins and related structures, fluorescent complexes or $\mathrm{Ru}(\mathrm{II})$ and $\operatorname{Ir}(\mathrm{III})$ [2].

$\mathrm{O}_{2}$ probes based on Pt-porphyrin dyes can be detected on standard fluorescence plate readers (Fig. $1 \mathrm{~F}$ ) which support time-resolved fluorescence mode (TRF). The latter provides high sensitivity ( $\mathrm{S}: \mathrm{B}$ ratio) and quantitative and accurate readout of $\mathrm{O}_{2}$ concentration due to lifetime-based detection mode. Modern instruments also allow active atmospheric control in microplate compartment $\left(\mathrm{O}_{2}, \mathrm{CO}_{2}\right)$ and simultaneous measurement of other functional readouts or cell-based assays. Extracellular probes are compatible with standard 2D cell culture and are actively used to measure cellular oxygen consumption (in samples sealed with oil), cell metabolism, drug toxicity and safety [1-3]. Intracellular probes are used to measure in situ oxygenation in cell monolayer and for the monitoring of metabolic responses of cells to drugs and other stimuli - in unsealed samples and in real time.

Last, but not least, live fluorescence microscopy platforms allow the analysis of $\mathrm{O}_{2}$ in complex and heterogeneous samples (e.g. micro-tissue such as multi-cellular spheroids and organoids), also for $\mathrm{O}_{2}$ analysis on a single cell level and high-resolution 2D and 3D mapping of tissue $\mathrm{O}_{2}$ concentration. Again, phosphorescence lifetime imaging microscopy (PLIM) provides fully quantitative $\mathrm{O}_{2}$ readout and stable $\mathrm{O}_{2}$ calibration, while intensity based microscopy is usually qualitative or semi-quantitative.

Thus, $\mathrm{O}_{2}$ sensor technology provides a set of powerful tools for life science and biomedical research.

\section{References}

[1] Papkovsky DB, Zhdanov AV, Phosphorescence based $\mathrm{O}_{2}$ sensors - Essential tools for monitoring cell and tissue oxygenation and its impact on metabolism - Free Rad Biol Med, 2016, 101: 202-210.

[2] Quenched-phosphorescence Detection of Molecular Oxygen: Applications in Life Sciences, Eds. DB Papkovsky, RI Dmitriev, RSC Detection Sciences, 2018, 9781788011754.

[3] Dmitriev RI, Papkovsky DB, Optical probes and techniques for $\mathrm{O}_{2}$ measurement in live cells and tissue. (Review), Cell Mol Life Sci, 2012, 69(12): 2025-2039. 